\title{
Network Modelling of 3-3 Piezocomposite Materials
}

\author{
R. W. C. LEWIS, A. C. DENT, AND C. R. BOWEN* \\ Materials Research Centre, Department of Mechanical Engineering, \\ University of Bath, Bath, BA2 7AY, UK
}

\begin{abstract}
Micromechanical models of 3-3 piezocomposites have previously relied on an idealised unit cell containing a single pore. This oversimplification has contributed to the poor agreement between experimental and model results. With increased computational power and more efficient modelling techniques, larger models can be created to represent a network structure with numerous randomly placed pores, filled with a secondary passive phase of air or polymer. Such models more closely represent a porous microstructure and enable the prediction of hydrostatic figures of merit. A series of large three-dimensional porous piezocomposite structures were modelled, with predicted values in good agreement with existing experimental results. In addition, modelling a large number of random porous microstructures revealed the variability in composite properties, as is found in practise.
\end{abstract}

Keywords Piezoelectric; composite; finite element modelling; 3-3 connectivity

\section{Introduction}

The applications of high density lead zirconate titanate (PZT) ceramic can occasionally be limited, for example when used as a sensor of low frequency hydrostatic waves in SONAR applications [1-3]. Research into this field has established that porous piezoelectric composite devices with a low-density and high porosity, can provide improved hydrostatic properties and high figures of merit [2]. In addition, porous piezoelectrics have particular advantages for use with low density media, such as water and biological tissue, where the device must be acoustically matched with the medium. In this case, if the second phase of the piezocomposite is of low density (polymer or air), better acoustic matching is achieved [4].

Biphasic piezocomposites with 3-3 connectivity have been found to be particularly suited to hydrostatic applications and offer particular advantages in terms of simple sample preparation [5]. The 3-3 connectivity pattern is typical of ceramic foams with interconnecting struts in three dimensions.

\section{Figures of Merit}

In the active mode the hydrostatic charge coefficient $\left(d_{h}\right)$ is used to define the hydrostatic strain per unit electric field or charge per unit hydrostatic force, and is related to the material properties parallel and transverse to the polarisation direction (both $d_{31}, d_{32}$ transversely

Received September 5, 2006; revised September 22, 2006; accepted September 25, 2006.

*Corresponding author. E-mail: c.r.bowen@bath.ac.uk. 
and $d_{33}$ in polarisation), as defined by Eq. 1 .

$$
d_{h}=d_{33}+\left(d_{31}+d_{32}\right)\left(\mathrm{mV}^{-1} \text { or } \mathrm{CN}^{-1}\right)
$$

In the case of a passive detector, hydrophone sensitivity is related to the piezoelectric voltage coefficient $\left(g_{h}\right)$, defined as the electric field generated per unit hydrostatic pressure. This is calculated by the quotient of $d_{h}$ and the constant stress permittivity $\left(\varepsilon_{33}^{T}\right)$, as shown in Eq. 2.

$$
g_{h}=\frac{d_{h}}{\varepsilon_{33}^{T}}\left(\mathrm{Vm}^{-1} \mathrm{~Pa}^{-1}\right)
$$

The hydrostatic figure of merit (HFOM), $d_{h} . g_{h}$, is the product of Eqns. 1 and 2 and is used to assess the properties of a device used in both active and passive roles, e.g. sonar and medical applications [6, 7].

Finite element and analytical modelling of piezocomposites have been extensively studied for many connectivity patterns (e.g. 1-3 composites $[1,2]$ ), however, there has been less research into 3-3 composites than other areas. Banno [8] derived models for 3-0 and 3-3 connectivity that used a single unit cell and single pore with 4 divisions based on the biphasic composite microstructure of Rittenmyer et al. [9], with theoretical findings not fully matching experimental results. Banno highlighted that these differences were related to the microstructure of the porous PZT, which was not fully represented by the unit cell model [8].

Due to the oversimplification of the 3-3 models that only consider a single pore, model outputs have differed from experimental results. In this paper a model is developed which better represents a porous PZT ceramic in which the porosity in impregnated with air or polymer. While earlier papers considered single pores $[2,8,9,11]$, this paper will use a three-dimensional network to model a PZT material containing a random array of a large number of pores at a predefined volume fraction of porosity. Porous piezoelectric materials are often manufactured by the addition of a polymer that is burnt out during the sintering processes. This manufacturing approach provides a variety of pore types and, depending of the volume fraction of porosity, the porosity is not always fully interconnected and can even be totally isolated. The intention of this work is to create three dimensional networks that contain isolated, partially interconnected and fully interconnected regions of porosity or PZT, so that their influence on material properties can be investigated.

\section{Network Modelling of 3-3 Composites}

When the piezocomposite is utilised as a hydrophone, a variety of physical properties must be taken into account. Figures of merit relevant to hydrophone/SONAR applications $\left(d_{h}, g_{h}\right.$ and $d_{h} . g_{h}$ ), are dependent on the physical properties of each phase (piezoelectric, air or polymer) and the microstructure (PZT volume fraction and distribution of porosity). These must therefore be taken into consideration whilst modelling the material.

The approach developed considers a large number of cells or grains arranged in a network to achieve a more realistic microstructure. Three-dimensional models were constructed with 30 cells per side (Fig. 1), since this resolution (27,000 cells) provided reliable results and reasonable computational economy. Each of the cells in the model was randomly assigned either the properties of the piezoelectric PZT phase or the passive second phase of the composite (polymer or air). The porosity/density of the model was modified by varying 


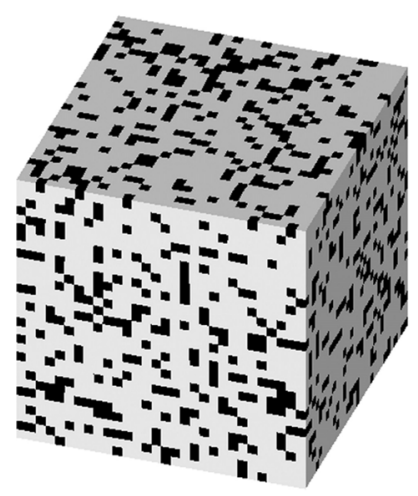

(a)

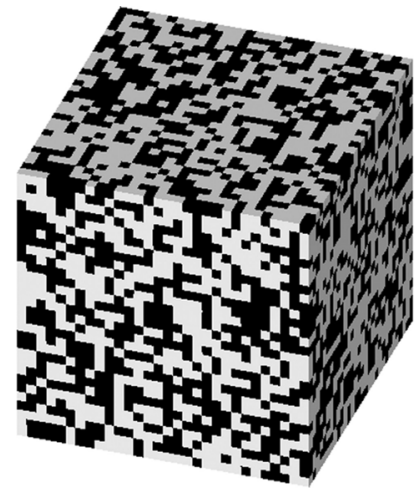

(b)

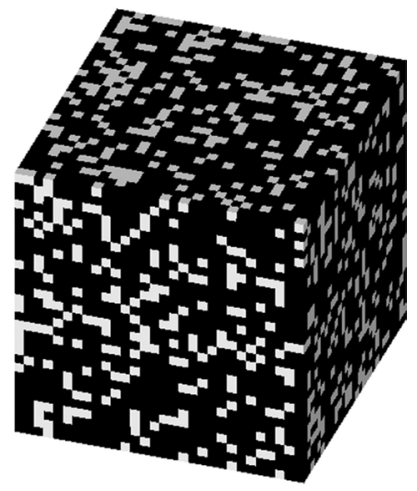

(c)

Figure 1. A characteristic model with a fine microstructure (30 grains/sides). The darker cells are PZT-5H, and lighter cells are the second phase material (air or polymer). (a) PZT-5H 25\% volume fraction, with (b) and (c) 50 and $75 \%$ respectively.

the ratio of the assigned cells respectively. Since the volume of modelled network was constant, a decrease in PZT volume fraction resulted in an increase in second phase cells.

The materials chosen to fill the porosity were air, polyethylene and epoxy, since these represent typical materials used in such composites and provide a range of compliances. PZT-5H was used as the piezoelectric material since it is a typical 'soft' PZT sensor material. Table 1 and 2 show the properties of the materials used to model the porous piezocomposite taking into account the isotropic and anisotropic properties of the materials.

Modelling was conducted with the commercial package ANSYS 8.1 using a coupledfield 8-node element (SOLID5), capable of the linear piezoelectric effect. Figure 1 illustrates three typical models with densities of 25,50 and $75 \%$ volume fraction of PZT-5H. The lighter cells are the piezoelectric ceramic (PZT-5H) and the darker cells are the second phase material (air or polymer). The voltage degrees of freedom were coupled for the nodes on the top and the bottom faces of the modelled block, to form two effective electrodes. An electric field was applied resulting in a displacement between the two faces (due to the piezoelectric effect). From the resulting displacement of the network in the $\mathrm{x}, \mathrm{y}$ and $\mathrm{z}$ directions, the strain per unit electric field and thus $d_{33}, d_{31}$ and $d_{32}$ was determined. From this data $d_{h}$ was calculated using Eq. 1 . By reading the charge developed on the model electrodes, the effective permittivity at constant stress $\varepsilon_{33}^{T}$ was determined, knowing the electrode separation and area. After calculating $d_{h}$ and $\varepsilon_{33}^{T}, g_{h}$ could be established using Eq. 2, along with the hydrostatic figure of merit, $d_{h} . g_{h}$. At each specific level of porosity and composition, over 20 individual random networks were evaluated to examine the typical

Table 1

Materials with anisotropic properties required for modelling of materials with ANSYS. Data provided by Dunn et al. [14]

\begin{tabular}{ccccccccccc}
\hline & $\mathrm{C}_{11}^{E}$ & $\mathrm{C}_{12}^{E}$ & $\mathrm{C}_{13}^{E}$ & $\mathrm{C}_{33}^{E}$ & $\mathrm{C}_{44}^{E}$ & $\mathrm{e}_{31}$ & $\mathrm{e}_{33}$ & $\mathrm{e}_{15}$ & \\
Material & $(\mathrm{GPa})$ & $(\mathrm{GPa})$ & $(\mathrm{GPa})$ & $(\mathrm{GPa})$ & $(\mathrm{GPa})$ & $\left(\mathrm{C} / \mathrm{m}^{2}\right)$ & $\mathrm{e}_{33}\left({\left.\mathrm{C} / \mathrm{m}^{2}\right)}^{\left(\mathrm{C}^{2} \mathrm{~m}^{2}\right)}\right.$ & $\mathrm{k}_{11}^{S} / \mathrm{k}_{0}$ & $\mathrm{k}_{33}^{S} / \mathrm{k}_{0}$ \\
\hline PZT-5H & 151 & 98.0 & 96.0 & 124 & 23.0 & -6.5 & 23.3 & 17.0 & 1700 & 1470 \\
\hline
\end{tabular}


Table 2

Materials with isotropic properties for modelling of materials with ANSYS. Data provided by Cutnell et al. [10]

\begin{tabular}{lccc}
\hline Material & $\varepsilon$ & $\begin{array}{c}\text { E } \\
(\mathrm{GPa})\end{array}$ & $\begin{array}{c}\text { Poisson's } \\
\text { Ratio }\end{array}$ \\
\hline Epoxy & 4 & 2.76 & 0.37 \\
Polyethylene & 4 & 0.73 & 0.46 \\
Air & 1 & 0 & 0 \\
\hline
\end{tabular}

scatter and variability of the predicted properties as a result of the stochastic distribution of porosity.

\section{Results and Discussions}

Figure 2 shows both $d_{33}$ and $d_{31}$ versus PZT volume fraction for PZT-air, PZT-polyethylene (PZT-PE) and PZT-epoxy composites. Each data point represents an individual model output, showing the scatter of results at each PZT fraction. At a specific PZT volume fraction, the $d_{33}$ coefficient increases when the stiffness of the passive second phase is reduced; for example, $d_{33}$ increases when changing from PZT-epoxy (highest stiffness) to PZT-air porosity (zero stiffness). Since porosity is randomly located in each model, variation between individual networks of the same porosity can be seen throughout the results. At the extremes of PZT volume fraction $(0 \%$ and $100 \%)$ there is no scatter in the model outputs, as would be expected, since each three-dimensional network is identical. This indicates that

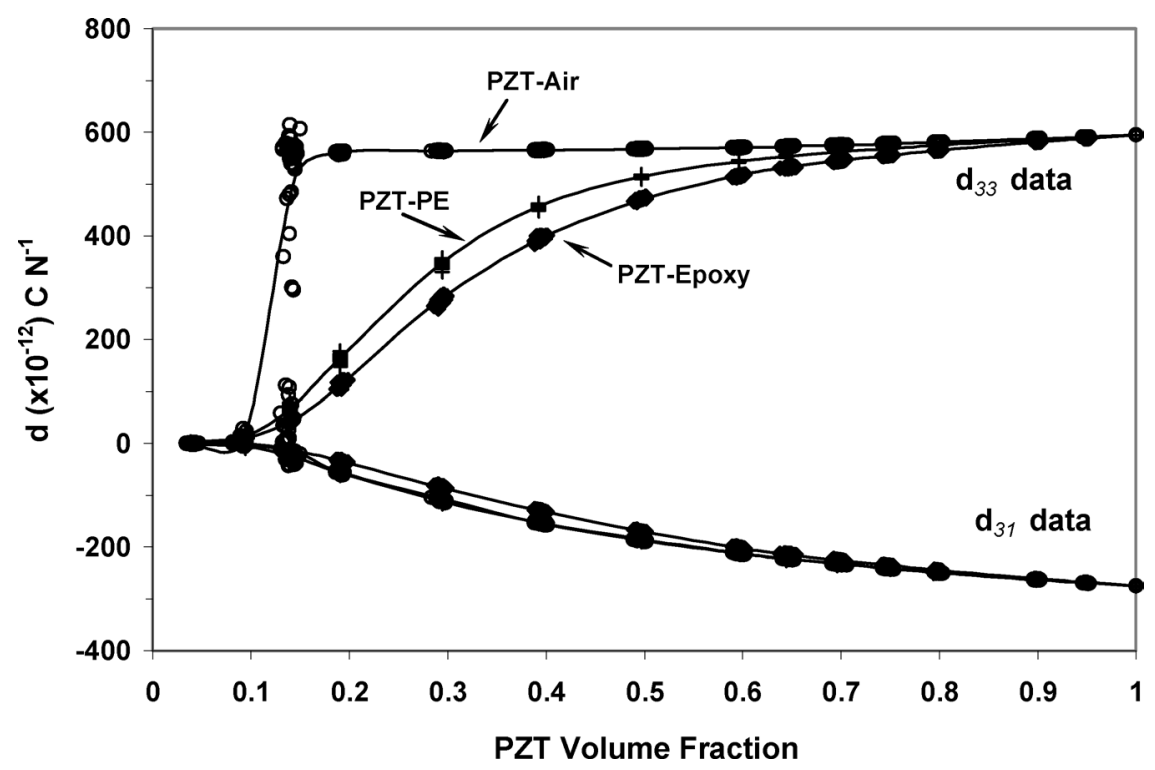

Figure 2. Variation of $d_{33}$ and $d_{31}$ with PZT volume fraction for PZT-air, PZT-polyethylene(PZT-PE) and PZT-epoxy. A decrease in $d_{33}$ and $d_{31}$ with increasing porosity is observed with significant scatter at low densities. 
the observed variability in results is purely due to the different arrangement of pores in each individual network and not an artefact of the modelling method.

As the PZT volume fraction is decreased from $100 \%$, a rapid reduction in $d_{33}$ for PZTair models occurs at $\sim 15 \%$ PZT, whereas the results for both PZT-polymer composites show a more gradual decrease. The rapid decrease in the PZT-air composite at $\sim 15 \% \mathrm{PZT}$ arises from the loss of any stress or strain transfer as the PZT cells loose connectivity and become isolated in a zero stiffness medium (air). In single pore models the $d_{33}$ remains constant to almost zero volume fractions of piezoelectric, since a single pore model cannot simulate the isolation of PZT in air $[2,8,11]$. The relatively large scatter of the $d_{33}$ results for PZT-air at $15 \%$ PZT is likely to be a result of some networks having some interconnection of PZT (high $d_{33}$ ), while other networks having purely isolated PZT (low $d_{33}$ ). For the PZT-air system with PZT volume fractions above $15 \%$ the $d_{33}$ is almost constant and close to the value of the fully dense piezoelectric material. This is not observed in experimental data [13] and may be due to the fact that while the piezoelectric cells in the model are fully poled and assigned the piezoelectric coefficients of Table 1, poling of a highly porous piezoceramic may be difficult and result in incomplete poling.

The $d_{31}$ coefficient values in Fig. 2 also decrease with a decrease in PZT volume fraction, and decline at a more rapid rate than that of $d_{33}$. Compared to $d_{33}$, the $d_{31}$ coefficient is less sensitive to the stiffness of the passive second phase. Due to the rapid decrease in $d_{31}$ and slow decrease in $d_{33}$, there is an increase in $d_{h}$ compared to the dense material (Fig. 3); as earlier papers have shown in depth $[1,9,12]$. For example, for a PZT-air composite the $d_{33}$ at $\sim 20 \%$ PZT is large while $d_{31}$ has significantly reduced (Fig. 2); resulting in high $d_{h}$. The $d_{h}$ falls at low PZT volume fractions $(<10 \%)$ due to decrease in $d_{33}$ of the piezocomposites in this volume fraction range.

Examination of Fig. 4 indicates that, as expected, the permittivity at constant stress $\varepsilon_{33}^{T}$ decreases with an increase of air or polymer, due to the decrease in the volume fraction of the high permittivity PZT material. Scatter between individual models outputs of permittivity at a specific PZT volume fraction is relatively low compared to the piezoelectric $d_{33}$ and $d_{31}$ coefficients (compare Figs. 2 and 4). When comparing the permittivity of PZT-air, PZTpolyethylene and PZT-epoxy materials in the PZT range of 25 to $80 \%$ (Fig. 4), a lower

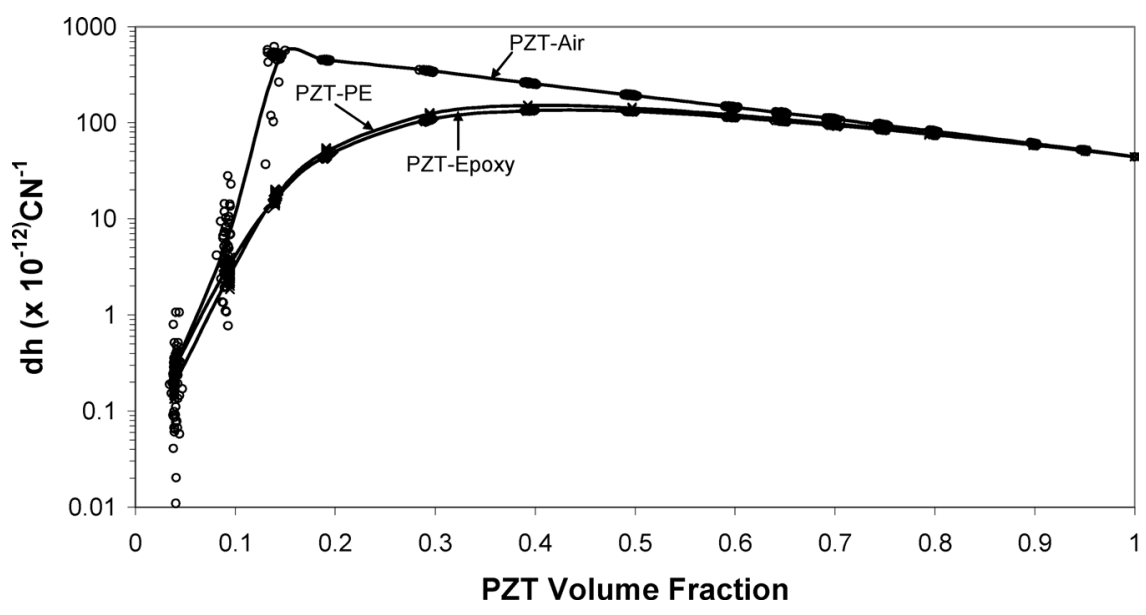

Figure 3. Variation of hydrostatic charge coefficient $d_{h}$ with PZT volume fraction for three PZTcomposites combinations. Scatter is found to increase at higher porosities $(\sim 15 \%)$. 


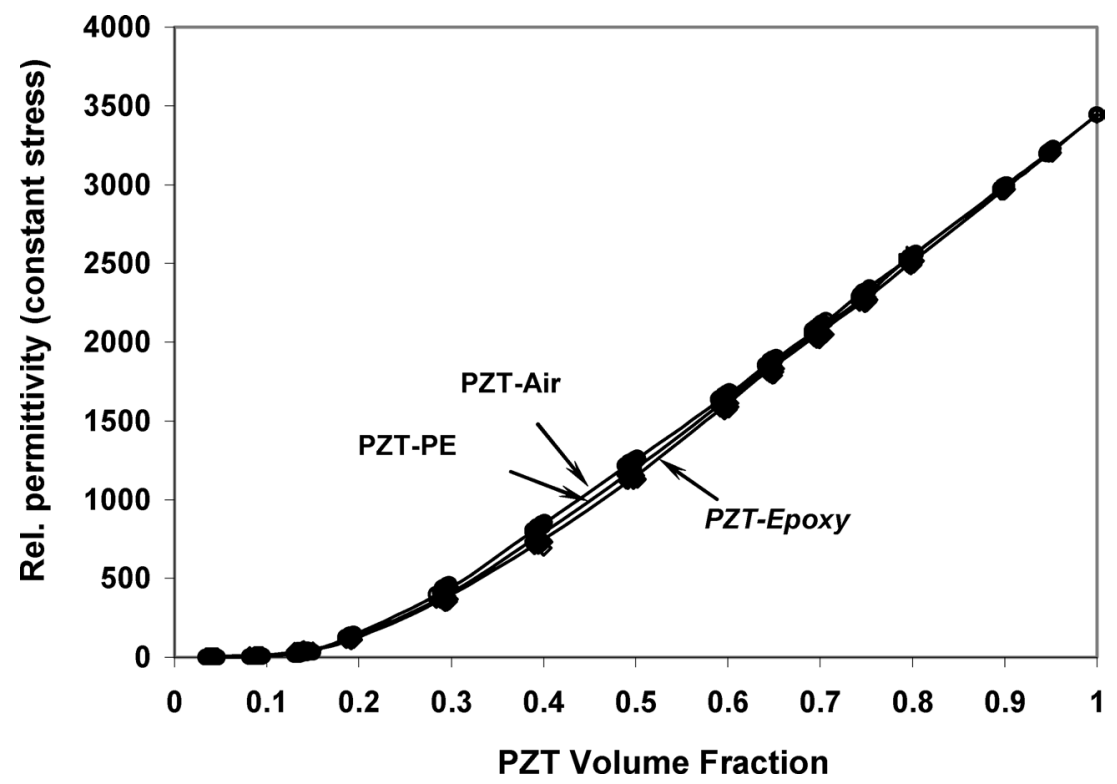

Figure 4. Variation of permittivity at constant stress $\varepsilon_{33}^{T}$ in the longitudial axis, showing decrease in permittivity as the passive phase (air or polymer) replaces the ferroelectric PZT material.

stiffness passive phase provides a slightly higher value of permittivity, possibly due to the higher $d_{33}$ of the materials which provides a contribution to $\varepsilon_{33}^{T}$. At high PZT volume fractions $(>20 \%)$ the dependency of permittivity of PZT volume fraction is almost linear, but falls to low values at PZT volume fractions below 15\%; again to the isolation of PZT cells in this range.

The estimation of $g_{h}$ from the data in Figs. 3 and 4 and using Eq. 2 is shown in Fig. 5, which shows there is a large increase in $g_{h}$ as the PZT volume fraction is reduced and the permittivity (Fig. 4) and $d_{31}$ is decreased (Fig. 2). The PZT-air materials exhibit the highest $g_{h}$ values due to their high $d_{33}$ and $g_{h}$ increases to a maximum at $\sim 15 \%$ PZT volume fraction. As with the $d_{h}$ data in Fig. 3, the decrease in $g_{h}$ at PZT volume fractions below $20 \%$ is a result of the PZT losing connectivity, resulting in low $d_{33}$ values (Fig. 2) and small $d_{h}$. Figure 5 shows a large scatter in $g_{h}$ is observed at PZT volume fraction at $15 \%$ and below. For example the mean $g_{h}$ at $15 \%$ PZT is $14.2 \times 10^{-3} \mathrm{Vm}^{-1} \mathrm{~Pa}^{-1}$, with a large standard deviation of $3.1 \times 10^{-3} \mathrm{Vm}^{-1} \mathrm{~Pa}^{-1}$. This is primarily due to the variability in the piezoelectric $d_{33}$ and $d_{31}$ coefficients in this PZT volume fraction range (Fig. 2).

Figure 6 shows $d_{h} . g_{h}$ as a function of PZT volume fraction for the PZT-air and PZTpolymer composites. At 50\% to 100\% PZT volume fraction the model output for the hydrostatic figure of merit are found to be in good agreement with published experimental data, which is also shown in Fig. 6. [2, 9, 12, 13]. While the models predict some scatter, the experimental data exhibits a higher degree of scatter which again may be associated with incomplete or variable poling of these porous materials.

Compared to PZT-air materials, results for PZT-polymer composites tend to show less scatter (see Figs. 2, 3, 5, and 6), mainly due to the fact that the second phase material is able to mechanically stabilise the porous structure and the non-zero stiffness enables strain transfer to occur. This is found to be in correlation with earlier models of the same material 


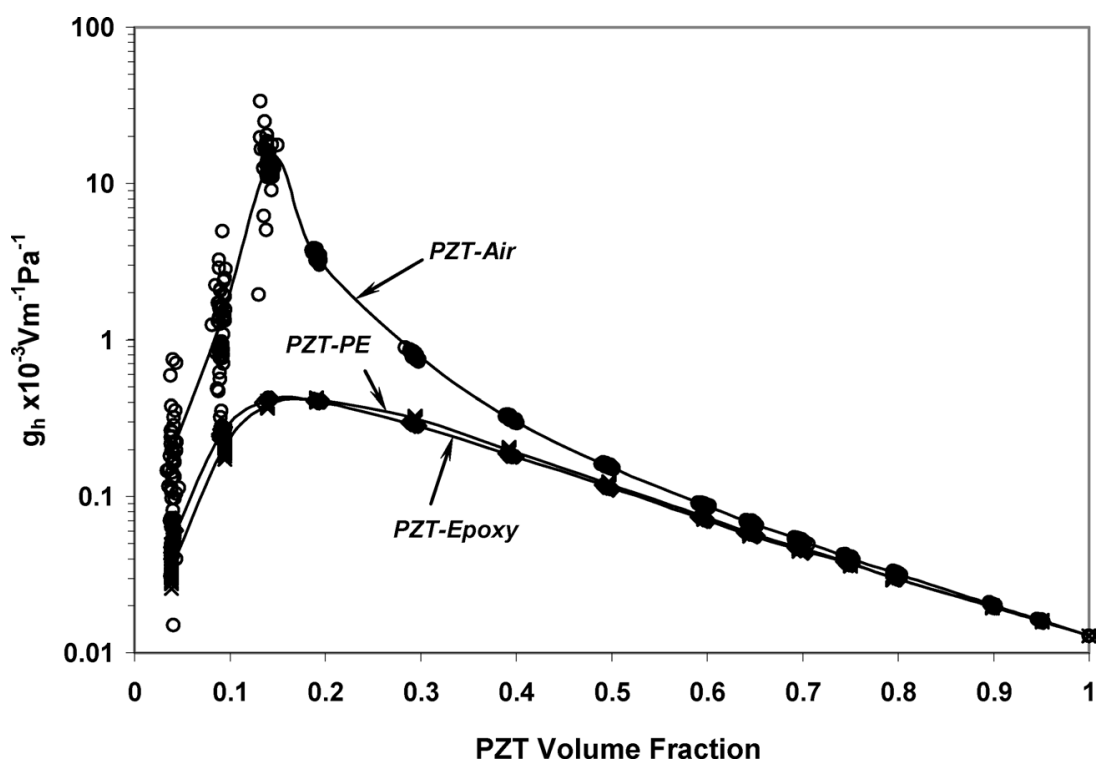

Figure 5. Piezoelectric voltage coefficient $g_{h}$ as a function of PZT volume fraction, which increases with decreasing PZT volume fraction due to a reduction in $\varepsilon_{33}^{\mathrm{T}}$ and $d_{31}$.

type [2]. From Fig. 6 the $d_{h} \cdot g_{h}$ hydrostatic figure of merit is at an optimum at PZT volume fraction of $\sim 30 \%$ and $d_{h} . g_{h}$ is greatly increased compared to that of monolithic dense PZT-5H. The piezocomposite $d_{h} \cdot g_{h}$ is found to increase by 50 times than that of the $100 \%$

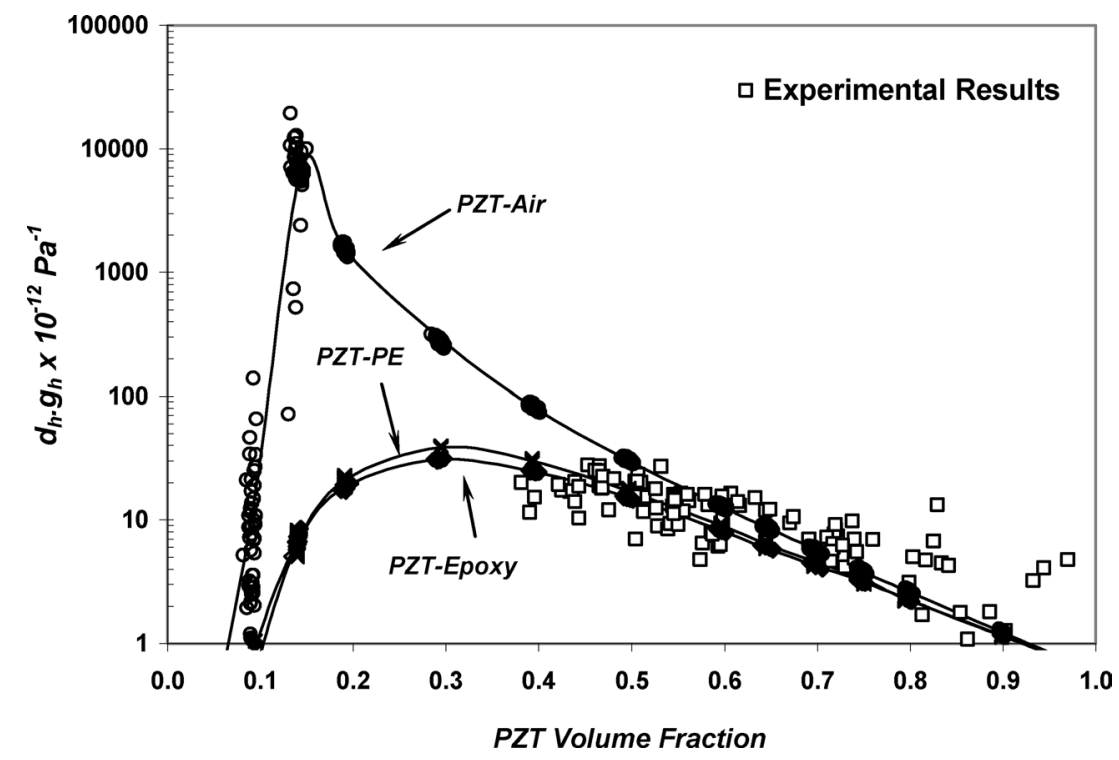

Figure 6. Hydrostatic figure of merit $\left(d_{h} \cdot g_{h}\right)$ as a function of PZT volume fraction, shows good correlation with experimental results (hollow symbols) [13]. 
dense PZT at 50\% density. As with $d_{h}$ and $g_{h}$, an increased stiffness of the passive phase decreases $d_{h} \cdot g_{h}$.

\section{Conclusions}

Three-dimensional networks have been developed to model the relevant hydrostatic figures of merit for porous PZT materials. Good correlation between model and experimental data has been observed with this technique. The model offers a number of advantages over current simplistic models $[2,8,9,11]$ which consider a single pore in a piezoceramic matrix. Principally, by modelling a random array of pores the change in properties as the composite system changes from fully interconnected PZT (high density) to fully isolated PZT (low density) can be modelled, as shown by $d_{33}$ results (see Fig. 2). Many of the single pore models are unable to model the decrease in $d_{33}$ and $d_{h}$ due to isolation of the PZT at low PZT volume fractions. In addition, by generating a large number of models of the same pore/polymer volume fraction the scatter of expected material properties can be evaluated due to differences in pore arrangement between each models. The models are more representative of the microstructure of real porous piezoelectric materials and could aid in composite selection to compromise between maximising the figure of merit for a particular application and minimising the potential variation of results between each sample or device. One limitation of the model is that the material is assumed to be fully poled in the polarisation direction, which may not be achievable in a highly porous PZT structure.

\section{Acknowledgments}

The authors acknowledge the EPSRC Piezo Device Modelling Network for support.

\section{References}

1. C. R. Bowen, A. Perry, H. Kara, and S. W. Mahon, Analytical modelling of 3-3 piezoelectric composites. J. Euro. Ceram. Soc. 21, 1463-1467 (2001).

2. H. Kara, A. Perry, R. Stevens, and C. R. Bowen, Modelling of 3-3 piezocomposites for hydrophones. British Ceramic Transactions 101, 139-142 (2002).

3. R. E. Newnham, A. Safari, J. Giniewicz, and B. H. Fox, Composite piezoelectric sensors. Ferroelectrics 60, 15-21 (1984).

4. S. Geis, J. Fricke, and P. Löbmann, Electrical properties of PZT aerogels. J. Euro. Ceram. Soc. 22 1155-1161 (2002).

5. R. E. Newnham, D. P. Skinner, K. A. Klicker, A. S. Bhalla, B. Hardiman, and T. R. Gururaja, Ferroelectric ceramic-plastic composites for piezoelectric and pyroelectric applications. Ferroelectrics 27, 49-55 (1980).

6. D. P. Skinner, R. E. Newnham, and L. E. Cross, Flexible composite transducers. Mat. Res. Bull. 13, 599-607 (1978).

7. H. Kara, R. Ramesh, R. Stevens, and C. R. Bowen, Porous PZT ceramics for receiving transducers. IEEE Transactions on Ultrasonics, Ferroelectrics, and Frequency Control 50, 289-296 (2003).

8. H. Banno, Effects of porosity on dielectric, elastic and electromechanical properties of $\mathrm{Pb}(\mathrm{Zr}$, Ti) $\mathrm{O}_{3}$ ceramics with open pores: A theoretical approach. Jpn. J. Appl. Phys. 32, 4214-4217 (1993).

9. K. Rittenmyer, T. Shrout, W. A. Schulze, and R.E. Newnham, Piezoelectric 3-3 composites. Ferroelectrics 41, 189-195 (1982).

10. J. D. Cutnell and Johnson, Physics (New York: Wiley, 1995), p. 315. 
11. A. Perry, C. R. Bowen, H. Kara, and S. Mahon, Modelling of 3-3 piezocomposites. Scripta Materialia 41, 1001-1007 (1999).

12. V. Yu. Topolov, S. V. Glushanin, and C. R. Bowen, Piezoelectric response of porous ceramic and composite materials based on $\mathrm{Pb}(\mathrm{Zr}, \mathrm{Ti}) \mathrm{O}_{3}$ : Experiment and modelling. Advances in Applied Ceramics 104, 300-305 (2005).

13. C. R. Bowen, A. Perry, A. C. F. Lewis, and H. Kara, Processing and properties of porous piezoelectric materials with high hydrostatic figures of merit. J. Euro. Ceram. Soc. 24, 541-545 (2004).

14. M. L. Dunn and M. Taya, Electromechanical properties of porous piezoelectric ceramics. J. Am. Ceram. Soc. 76, 1697-1706 (1993). 